\title{
HPV16 E6 polymorphism and physical state of viral genome in relation to the risk of cervical cancer in women from the south of Poland*
}

\author{
Slawa Szostek ${ }^{\bowtie 凶}$, Barbara Zawilinska1, Malgorzata Klimek² and Magdalena Kosz-Vnenchak \\ 1Department of Virology, Chair of Microbiology, Jagiellonian University Medical College, Kraków, Poland; ${ }^{2}$ Department of Gynecological Oncol- \\ ogy, Maria Skłodowska-Curie Memorial Cancer Centre and Institute of Oncology, Cracow Branch, Kraków, Poland
}

\begin{abstract}
The aim of this study was to analyse the correlation between HPV16 E6 variants and the physical status of viral genome (integrated, mixed, episomal) among patients with cervical cancer $(n=40)$ and low-grade squamous intraepithelial lesions - LSIL $(n=40)$. The study was performed on 80 HPV16 positive samples. HPV16 E6 variants were identified using PCR and DNA sequencing. Nucleotide sequences of E6 were compared with the prototype sequence (EUR-350T). The physical state of HPV DNA was determined as the ratio of E2/E6 copy number per cell. Twelve different intratypic variants were identified as belonging to European (in 77 samples) and North-American 1 (in 3 samples) sublineages. The most prevalent non-synonymous variant was EUR-350G, which occurred with similar frequency in cervical cancer and LSIL. The frequencies of additional mutations in variants with EUR-350T or EUR-350G sequences differed significantly. For the first time, missense mutations G122A, C153T and G188A were discovered in EUR-350G variant. The integrated viral genome was predominant in women with cervical cancer. The EUR-350T prototype and EUR350G without additional mutations variants were prevalent in cervical cancer samples with the HPV16 characterized by integrated DNA. In summary, European variants of HPV16 E6 dominated in both cancer and LSIL group. The presence of EUR-350G favoured the occurrence of additional nucleotide changes. We showed that nucleotide changes occur significantly more often in the mixed form of viral DNA and in LSIL group and that the variants without additional mutations may promote integration of HPV16 genome.
\end{abstract}

Key words: HPV type 16 E6 variants, HPV16 physical state, real-time $P C R$, squamous intraepithelial lesions, invasive cervical carcinoma

Received: 07 June, 2016; revised: 24 August, 2016; accepted: 19 October, 2016; available on-line: 02 November, 2016

e-mail: sszostek@cm-uj.krakow.pl

*New nucleotide data was submitted and deposited in the GenBank databases and an accession numbers are KX462887, KX462888, KX462889

Abbreviations: HPV human papillomavirus; LSIL, low-grade squamous intraepithelial lesions; FIGO, International Federation of Gynaecology and Obstetrics; qPCR, quantitative real-time PCR

\section{INTRODUCTION}

Human papillomavirus type 16 (HPV16) has been classified into Alphapapillomavirus-9 species belonging to Papillomaviridae family. It is the most common type detected in early and malignant cervical lesions in the world as well as in some HPV-related cancers of the vulva, vagina, penis, anus, and oropharynx (Crow, 2012; Garbuglia, 2014; Bruni et al., 2015).

Persistent infection with oncogenic human papillomavirus genotypes is essential, although not sufficient, to cause invasive cervical cancer and cervical intraepithelial neoplasia as its precursor (Walboomers et al., 1999; Munoz et al., 2003).

The distribution of HPV genotypes in various populations and areas is different and the epidemiological studies suggest that certain HPV16 E6 variants could influence the persistence of infection, progression to precancer and cancer development (Londesborough et al., 1996). A distinct HPV type is confirmed when the DNA sequence of the L1 ORF of the cloned viral genome differs from that of any other characterised type by at least $10 \%$ but isolates of the same HPV type are named as subtypes when the nucleotide sequences of the L1 ORF differ by less than 10\% (de Villiers et al., 2004; Bernard et al., 2010). Cornet and coworkers (2012) proposed to define major variant lineages based on approximately $1.0 \%$ difference between full genomes of the same HPV type while variant sublineages by $0.5-0.9 \%$ differences. Variants of E6 HPV16 were divided into four distinct phylogenetic branches, the distribution of which varies geographically: lineage A - European-Asian, including the sublineages European (EUR) and Asian (As); lineage B - African 1 (Afr1) with Afr1a and Afr1b sublineages; lineage C - African 2 (Afr2) including Afr2a and Afr2b sublineages and lineage D - Asian American/North American including Asian American 1, Asian American 2 and North American 1 sublineages (Burk, 2013, Cornet et al., 2012).

Lee and coworkers (2008) proposed that nucleotide polymorphism within the E6 sequence of HPV16, rather than in other ORFs, influences the progression of cervical malignancy, and that additional factors are likely to play a significant role as well.

Integration of viral DNA into host cell genome is a frequent event in carcinogenesis. The HPV genome integration usually disrupts E2 gene, leading to overexpression of E6 and E7 oncogenes.

The aim of the present study was to identify HPV 16 E6 variants and to analyse the physical status of HPV16 genome (integrated and/or episomal form of viral DNA) among patients with cervical cancer in comparison to women with low-grade squamous intraepithelial lesions (LSIL). 


\section{MATERIALS AND METHODS}

Specimens and DNA extraction. The study was performed on eighty HPV16 positive cervical cytobrush samples confirmed by the INNO-LiPA HPV genotyping assay (Innogenetics). All HPV isolates included in the present study came from the collection of samples obtained from women (aged 22-90 years, mean 42 115 ) with invasive cervical carcinoma $(n=40)$ and low-grade squamous intraepithelial lesions - LSIL $(n=40)$. The LSIL group was dominated by younger women (up to 40 years of age) and women over the age of 50 were predominant in the cervical cancer group $(\phi<0.001)$. All samples were taken after cytological examination, but before any treatment. All cancer patients were treated at the Maria Skłodowska-Curie Memorial Cancer Centre and Institute of Oncology, Cracow Branch, Poland. The study was approved by the Ethics Committee of the Jagiellonian University.

DNA was isolated from cervical smears and purified using Genomic DNA Prep Plus kit (A\&A Biotechnology, Poland) according to the manufacturer's instructions.

HPV 16 E6 variants identification. Determination of HPV16 E6 variants was performed using PCR with type-specific E6 primers according to Zuna and coworkers (2009) and followed by DNA sequencing.

The final PCR reaction contained $50 \mathrm{ng}$ of the isolated DNA, 1x PCR Buffer II (Applied Biosystems, USA), $2 \mathrm{mM} \mathrm{MgCl}, 200 \mu \mathrm{M}$ dNTPs, $2 \mu \mathrm{M}$ each primer and 1 U AmpliTaq Gold DNA polymerase (Applied Biosyste$\mathrm{ms}$, USA). The specific primers, flanking the encoding E6 open reading frame (nt 83 - 559) comprised: 5' TGA ACC GAA ACC GGT TAG TA - 3' and 5' CAT GCA ATG TAG GTG TAT CTC C - 3'. The PCR conditions were as follows: polymerase activation $3 \mathrm{~min}$ at $94^{\circ} \mathrm{C}, 40$ cycles of denaturation at $94^{\circ} \mathrm{C}$ for 1 min, annealing at $55^{\circ} \mathrm{C}$ for $1 \mathrm{~min}$, and extension at $72^{\circ} \mathrm{C}$ for $2 \mathrm{~min}$, and a final elongation at $72^{\circ} \mathrm{C}$ for $7 \mathrm{~min}$. For every reaction, positive control using DNA extracted from $\mathrm{SiHa}$ cell line, as well as a negative control without template DNA $\left(\mathrm{H}_{2} \mathrm{O}\right)$, were included.

The amplified DNA fragments were tested by electrophoresis in $2 \%$ agarose gel with ethidium bromide. The PCR products were sequenced by Genomed (Poland).

The ExoSAP-IT clean up kit (USB, USA) was used to purify these products and DNA sequences were obtained with an ABI 3730xl DNA Analyzer or 3130xl Genetic Analyzer (Applied Biosystems, USA) using the same primers as those used for PCR and BigDye ${ }^{\mathrm{TM}}$ Terminator V3.1 Cycle Sequencing Kits (Applied Biosyste$\mathrm{ms}$, USA). The same forward and reverse primers were used separately in cycle sequencing in order to sequence both sense and antisense strands.

The obtained HPV16 E6 sequences were aligned with those of European prototype of HPV16 sequence (GenBank accession numbers: K02718, NC_001526), and compared with those of known HPV types available through the GenBank database (NCBI, National Institute of Health, Bethesda, MD, USA), using the BLAST 2.0 and ChromasPro 1.5 software.

HPV16 physical status determination. The method of the viral physical state assessment as a ratio of E2 to E6 copy numbers per cell was described in our previous paper (Szostek et al. 2015). Amplification was performed using the ABI Prism 7500 Fast Real-Time PCR Systems for HPV16 E6 and E2 sequences. The quantity of E2 and E6 genes was detected simultaneously by multiplex quantitative PCR. The load of E2 and E6 was determined using a copy number normalized per cell.
The number of cells was assessed by qPCR targeting the RNase P open reading frame. The E2/E6 ratios were calculated to determine the pure episomal (E2/EG $\geq 1.0)$, the mix of integrated and episomal $(0.99>E 2 / E 6>0.05)$ and the integrated $(E 2 / E 6 \leq 0.05)$ forms present in a single sample.

Statistical analysis. The statistical analysis was performed using the STATA 10.0 software package. Descriptive statistics were used to determine mean value of continuous variables and standard errors of means. Correlations between $\mathrm{Ct}$ levels were analysed by correlation matrix in which $\mathrm{R}$ coefficients and $\mathrm{p}$ values were calculated. The distribution of HPV16 variants in the investigated groups and in different physical status forms of viral DNA was described using descriptive statistics and non-parametric Mann-Whitney $U$ test for the number of nucleotide changes in the analysed sequences. Dichotomous variables were analysed using the chi-square test. A p-value less than 0.05 was considered significant. A multivariate logistic regression analysis was used for assessing the relationship between clinical diagnosis and the occurrence of nucleotide changes.

\section{RESULTS}

\section{HPV16 E6 variants detection}

Nucleotide sequences of the complete E6 ORF from 80 patients were compared with the HPV16 E6 prototype sequence (GenBank KO2718). Table 1 shows variant analysis for the E6 gene. A total of 12 different intratypic variants were identified and were classified into two different lineages (A and D), including European (EUR) and the North-American 1 (NA1) sublineages.

Among the sequences belonging to the European sublineage, 26 out of $77(34 \%)$ were identical with the prototype sequence (EUR-350T). Moreover, there were only 3 samples with the EUR-350T sequence with an additional single nucleotide changes, at position T137G $(n=2)$, resulting in amino acid change $(\mathrm{L} 12 \mathrm{~V})$, and at T109C without amino acid change (silent mutation), in one case. EUR-350T was present in 32.5\% (13/40) of cervical cancer samples and in $16 / 40(40 \%)$ of the LSIL group, but the differences were not statistically significant. The EUR-350G variants were the most frequently detected isolates in our population and represented $60 \%$ $(48 / 80)$ of total HPV16 samples, with similar frequency in cervical cancer and LSIL groups. The NA1 sublineage was detected in $4 \%(3 / 80)$ of samples, only in the cervical cancer group. Distribution of HPV16 E6 variants according to different sublineages in LSIL and carcinoma groups was not statistically different (Pearson's chi-squared, $p=0.19)$.

Sequence analysis of 80 studied samples showed substitutions in 9 nucleotides located between positions 122 and 350 (G122A, T137G, G145T, C153T, G176A, G188A, T295G, C335T, T350G) leading to the amino acid change (E7K, L12V,Q14H, T17I, D25N, E29K, D64E, H78Y, L83V) while other nucleotide substitutions (T109C, C256T, T286A, A289G) were silent. Moreover, for the first time, unknown missense mutations G122A, C153T and G188A were recorded along with EUR-350G (Table 1). Only in two cases, the amino acid changes (Q14H and T17I) were non-conservative, while other changes were conservative. The most prevalent non-synonymous variant was L83V (T350G). It was found in 64\% (51/80) of samples in both the European and NA1 sublineages, 
Table 1. HPV16 E6 variants and their lineage identified in 80 patients with cervical cancer and low-grade squamous intraepithelial lesions.

Capital letters indicate variants with an amino acid change, while small letters indicate silent mutation; at the bottom of the table amino acid changes are marked; $\mathrm{N}^{*}$ - total cases; LSIL - low-grade squamous intraepithelial lesions; ICC - invasive cervical cancer; AA - amino acid.

\begin{tabular}{|c|c|c|c|c|c|c|c|c|c|c|c|c|c|c|c|c|c|c|}
\hline & & $\begin{array}{l}\text { Nucleotide } \\
\text { position }\end{array}$ & 109 & 122 & 137 & 145 & 153 & 176 & 188 & 256 & 286 & 289 & 295 & 335 & 350 & & & \\
\hline & & $\begin{array}{l}\text { HPV16 } \\
\text { prototype }\end{array}$ & $\mathrm{T}$ & G & $\mathrm{T}$ & G & $C$ & G & G & C & $\mathrm{T}$ & $A$ & $\mathrm{~T}$ & C & $\mathrm{T}$ & & & \\
\hline $\begin{array}{l}\text { Line- } \\
\text { age }\end{array}$ & $\begin{array}{l}\text { Subline- } \\
\text { age }\end{array}$ & Class/subclass & & & & & & & & & & & & & & $\mathrm{N}^{*}=80$ & $\mathrm{LSIL}=40$ & $I C C=40$ \\
\hline \multirow[t]{11}{*}{$A$} & EUR-p & EUR-350T & - & - & - & - & - & - & - & - & - & - & - & - & - & 26 & 13 & 13 \\
\hline & & EUR-350T/137G & - & - & $\mathrm{G}$ & - & - & - & - & - & - & - & - & - & - & 2 & 2 & 0 \\
\hline & & EUR-350T/109C & C & - & - & - & - & - & - & - & - & - & - & - & - & 1 & 1 & 0 \\
\hline & EUR-350G & EUR-350G & - & - & - & - & - & - & - & - & - & - & - & - & G & 24 & 8 & 16 \\
\hline & & EUR-350G/109C & c & - & - & - & - & - & - & - & - & - & - & - & G & 12 & 9 & 3 \\
\hline & & EUR-350G/122A & - & A & - & - & - & - & - & - & - & - & - & - & G & 2 & 2 & 0 \\
\hline & & EUR-350G/153T & - & - & - & - & $\mathrm{T}$ & - & - & - & - & - & - & - & G & 1 & 1 & 0 \\
\hline & & EUR-350G/176A & - & - & - & - & - & A & - & - & - & - & - & - & G & 1 & 0 & 1 \\
\hline & & EUR-350G/188A & - & - & - & - & - & - & A & - & - & - & - & - & G & 1 & 0 & 1 \\
\hline & & EUR-350G/256T & - & - & - & - & - & - & - & $\mathrm{t}$ & - & - & - & - & G & 4 & 4 & 0 \\
\hline & & EUR-350G/295G & - & - & - & - & - & - & - & - & - & - & G & - & G & 3 & 0 & 3 \\
\hline \multirow[t]{4}{*}{ D } & NA1 & & - & - & - & $\mathrm{T}$ & - & - & - & - & a & $\mathrm{g}$ & - & $\mathrm{T}$ & G & 3 & 0 & 3 \\
\hline & & Codon position & & 7 & 12 & 14 & 17 & 25 & 29 & & & & 64 & 78 & 83 & & & \\
\hline & & Reference AA & & $E$ & $\mathrm{~L}$ & Q & $\mathrm{T}$ & D & $E$ & & & & $\mathrm{D}$ & $\mathrm{H}$ & $\mathrm{L}$ & & & \\
\hline & & Mutated AA & & $\mathrm{K}$ & V & $\mathrm{H}$ & 1 & $\mathrm{~N}$ & $\mathrm{~K}$ & & & & $E$ & $\mathrm{Y}$ & V & & & \\
\hline
\end{tabular}

either alone $(47 \%, 24 / 51)$ or along with other nucleotide changes $(53 \%, 27 / 51)$. The prevalence of T350G was similar in cervical cancer and LSIL groups and amounted to $67.5 \%$ and $60 \%$, respectively (Pearson's chi-squared, $p=0.48)$. Missense mutations in nucleotides other than EUR-350G were detected in individual samples. Among the synonymous variants T109C dominated $(16 \%, 13 / 80)$.

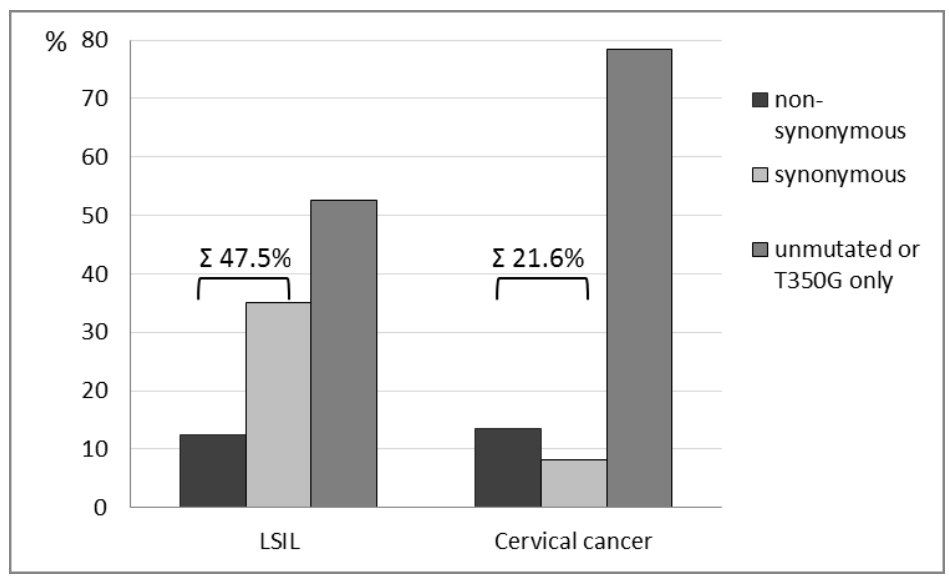

Figure 1. Distribution of additional nucleotide changes (without T350G) in the European variants of samples with LSIL and cervical carcinoma taking into account synonymous and non-synonymous mutations.

The differences of mutations number between groups were significant (Pearson's chi-squared, $p=0.02$ ).
In samples of the European lineage, frequencies of additional mutations (synonymous and non-synonymous) in variants with EUR-350T or EUR-350G sequences differed significantly and occurred in 10\% (3/29) and 50\% $(24 / 48)$ of samples, respectively (Pearson's chi-squared, $p=0.0004$ ). Furthermore, additional mutations (excluding T350G) in these samples were observed more often in LSIL than in cervical cancer, in $47.5 \%$ and $21.6 \%$ respectively (Pearson's chi-squared, $p=0.02$ ) (Fig. 1). The number of all mutations, including three missense mutations and two silent ones, calculated per sample was highest in the NA1 sublineage variant. In European variants, only one or two nucleotide changes were observed. The average number of mutations per sample was not statistically significant when comparing cancer and LSIL patients (Mann-Whitney $U$ test, $p=0.29$ ). However, silent mutations were pointed out in the LSIL group significantly more often (Pearson's chi-squared, $p=0.003$ ) as shown in Fig. 1. A multivariate logistic regression analysis showed that the presence of synonymous mutations was correlated with a six-fold (OR 6.1; $95 \%$ CI 1.55 - 24.00; $p=0.003)$ increase in the probability of the occurrence of the LSIL diagnosis. The occurrence of mutation for the entire group $(\mathrm{N}=80)$ did not correlate with women's age (Mann-Whitney U test, $p=0.84)$. 


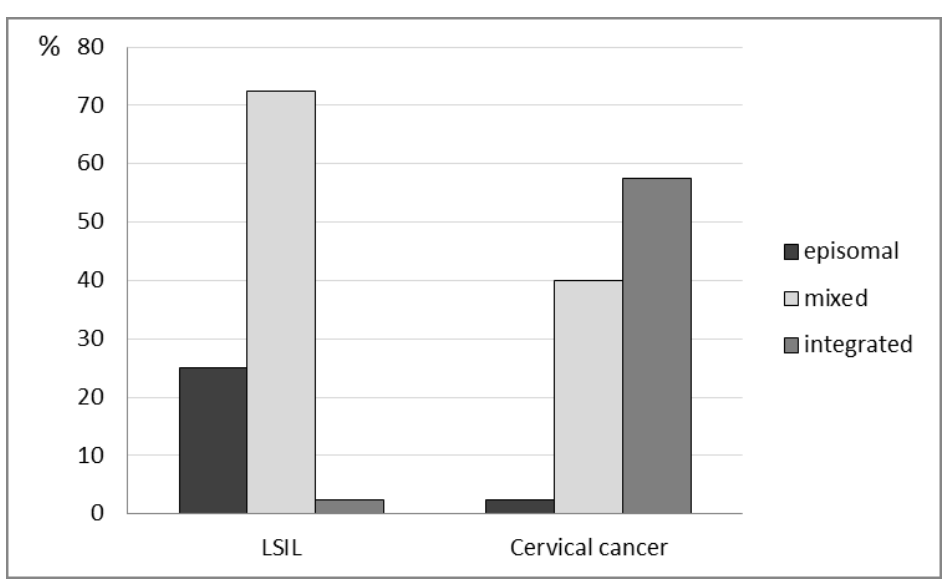

Figure 2. Physical state of HPV16 in relation to the studied groups $(n=40$ LSIL; $n=40$ cervical cancer).

The differences between groups were significant (Pearson's chi-squared 26.9 $(\mathrm{df}=2), p<0.0001)$.

\section{Detection of HPV16 physical status}

The integrated HPV16 DNA was identified in 24 $(30 \%)$, mixed form (episomal and integrated in the same sample) in $45(56.3 \%)$ and episomal form in 11 $(13.7 \%)$ samples. Physical status of HPV16 genome related to the studied groups (cervical cancer and LSIL) was significant (Fig. 2). The integrated and mixed forms of HPV16 DNA were statistically more often found in cervical cancer than in the LSIL group (48.7\% vs $37.5 \%$ respectively; V-chi-squared test, $p=0.004)$. Among the isolates containing the episomal form of HPV16 DNA, 91\% were defined as LSIL.

In women with LSIL, the episomal form of HPV16 DNA was present in $25 \%$, while the mixed form in $72 \%$ of cases. Only one material $(2.5 \%)$ was demonstrated to have integrated HPV16 genome. The integrated viral genome was predominant in women with cervical cancer $(23 / 40,57.5 \%)$. The mixed form of HPV16 DNA was found in $40 \%$ of women with cancer, whereas the episomal form was shown only in one patient.

\section{Association of HPV16 E6 polymorphisms with viral genome physical state}

In NA1 variant mixed form of HPV16 DNA was identified. Figure 3 shows occurrence of EUR-350T and EUR-350G variants in relation to the physical status of HPV16 DNA. The EUR-350G variant dominated

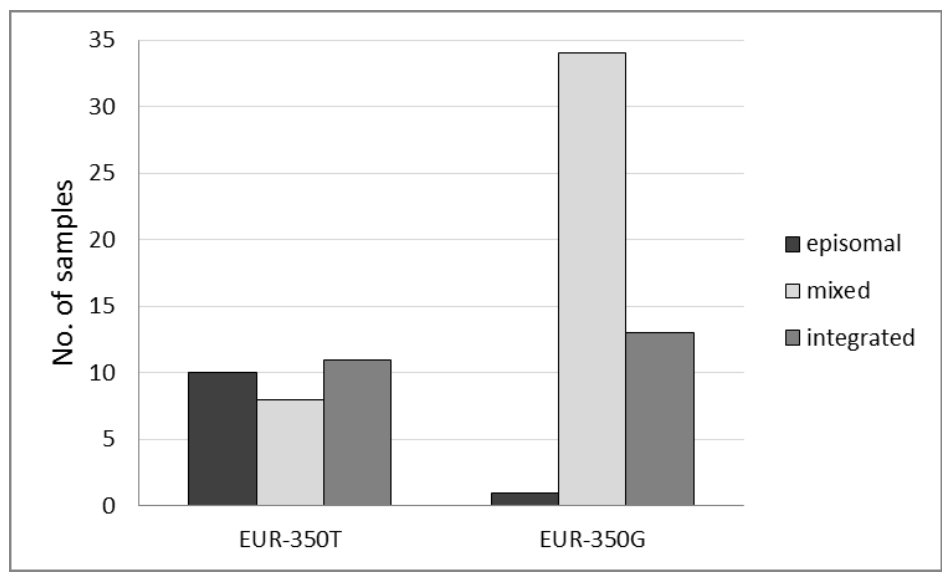

Figure 3. Physical state of HPV16 genome (episomal, mixed, integrated forms) in EUR-350T and EUR-350G variants. in mixed form while EUR-350T was slightly more often present as the integrated form. These differences were statistically significant (Pearson's chi-squared test, df $=2, p<0.0001$ ).

As regards the European variant, all mutations (non-synonymous as well as synonymous) were most frequently detected in mixed form compared to integrated form, $68.63 \%(35 / 51)$ and $25.49 \%(13 / 51)$, respectively. Only 3 samples with episomal forms of HPV16 genome contained the nucleotide changes (Pearson's chi-squared test, $p=0.0007$ ). In multivariate analysis, the distribution of the different variants depending on the form of the HPV16 genome and cytological diagnosis showed that in the samples of cervical cancer with the integrated form, EUR-350T prototype and EUR-350G without additional mutations were the most frequently observed. These results were statistically significant and amounted to, respectively: 43\% (10/23); Pearson's chi-squared 13.1 $(\mathrm{df}=2), p=0.001$ and $47 \%(11 / 23)$; Pearson's chi-squared $10.9(\mathrm{~d} f=2), p=0.004$. For the other variants, there was no such relationship. Figure 4 demonstrates the average number of nucleotide changes present in materials assigned to particular groups depending on the form of HPV16 genome. The percentage of synonymous and non-synonymous mutations in three forms of HPV16 genome was shown in Fig. 5.

Among samples with the episomal form, isolates in which the prototype sequence EUR-350T was detected were predominant $(72.7 \%, 8 / 11)$, while non-synonymous mutations usually were observed in the mixed and integrated forms, $34 / 42$ and $13 / 24$, respectively. These differences were statistically significant (Pearson's chisquared test, $p=0.002$ ).

\section{DISCUSSION}

HPV16 is the most commonly encountered high risk type of HPV in Polish women with cervical cancer. Diversity of the HPV16 E6 sequence was investigated in women from southern Poland. For the first time, in the present study, we also analysed the relationship between the polymorphism of HPV16 E6 gene and the viral genomic physical status in this patient group. It is widely accepted that the distribution of HPV variants is related to geographical or population ethnicity distribution (Xi et al., 2006; de Araujo Souza et al., 2009; Cornet et al., 2013a). In the present study, of 80 HPV16 positive women, European lineage variants (both prototype and related variant), were dominant $(96 \%)$ in both LSIL and cervical cancer group. In $3(4 \%)$ cases, only in the cervical cancer group, North American sublineage was detected. Similar results were obtained in Slovenian patients with cervical cancer, where mainly European genomic variations of HPV16 were found and only 2/40 (5\%) of the cases were associated with non-European HPV16 genomic variants - the AsianAmerican and African 2 branches (Vrtačnik Bokal et al., 2010). Several studies showed that non-European HPV16 genomic variants (lineage B, C, D) are associated with stronger oncogenic potential than the European line- 


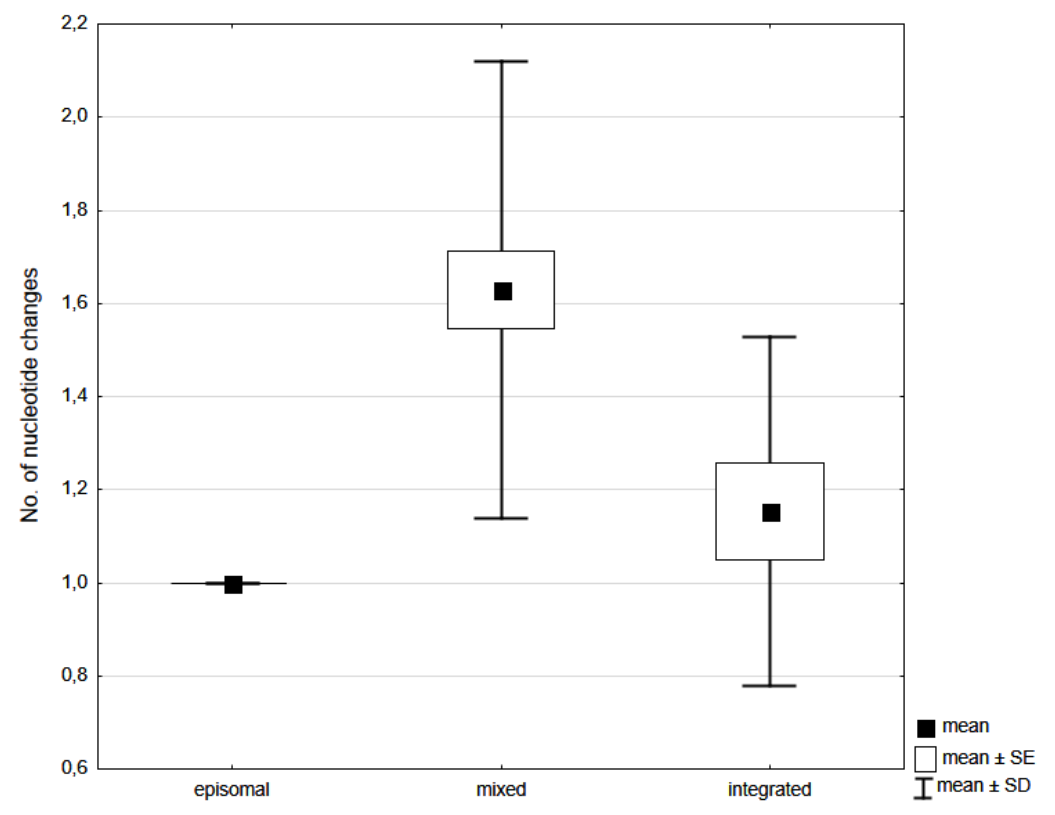

Figure 4. The average number of all nucleotide changes in European variants E6 sequence depending on the HPV16 physical status (episomal, mixed, integrated forms).

age A (Hildescheim et al., 2001; Xi et al., 2007; Sichero et al., 2007; Schiffman et al., 2010; Freitas et al., 2014; Ortiz-Ortiz et al., 2015). Moreover, the Asian American// North American (AA/NA ) variants were linked to higher capacity of in vitro transformation of human keratinocytes (Sichero et al., 2012).

On the other hand, studies performed in Sweden, Germany and the Netherlands failed to confirm such relationship between European and non-European lineages (Zehbe et al., 1998; Nindl et al., 1999; van Duin et al., 2000). However, almost 20 years ago, Yamada and coworkers (1997) analysed HPV16 sequence variation in a worldwide collection of cervical cancer specimens and showed that European lineage variants predominated in Europe and North America and a single North-American 1 variant was detected only in Argentina. While in recent research by Cornet and coworkers (2013a), it was suggested that there is an overrepresentation of AA/NA variants in cervical cancer cases in South/Central Ameri$\mathrm{ca}$ and shown that in Europe these variants are the most prevalent non-European variants (9/281, 3\%). Also,

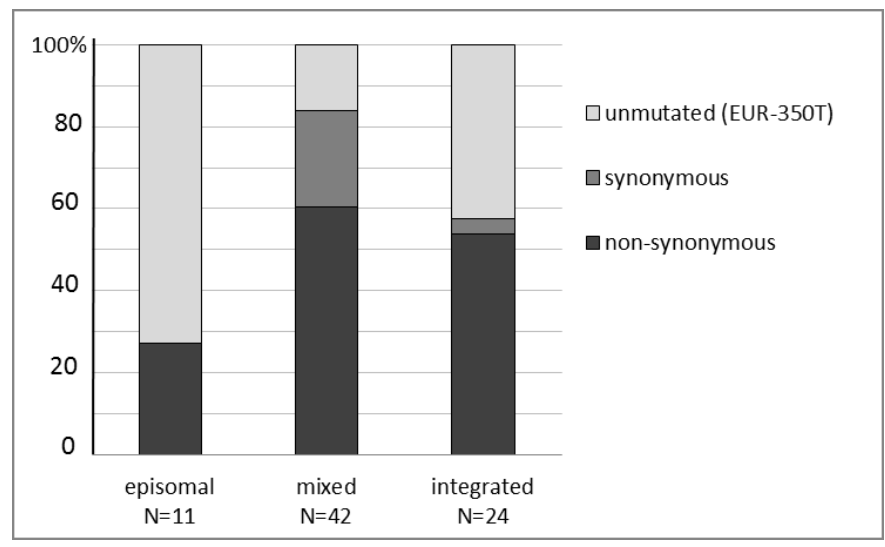

Figure 5. Distribution of synonymous and non-synonymous mutations in European variants of HPV16 E6 sequence depending on the form of viral genome. both Burroni and coworkers (2013) in Italian women with LSIL and Gudleviciene and coworkers (2015) in Lithuanian women with cervical cancer revealed a single case of the North-American 1 variant, which is similar to our findings in the population of women from the south of Poland.

The most common European polymorphism in E6 gene is a nucleotide substitution at position 350 (350G instead of $350 \mathrm{~T}$ ) that leads to a protein change in codon 83 , leucine to valine $(\mathrm{L} 83 \mathrm{~V})$. Notably, the same E-350G nucleotide variation is also a characteristic finding in all AsianAmerican and North-American sequences (Zuna et al., 2009). In some previous studies in Danish, Swedish and French populations, it was shown that this substitution is associated with an increased risk of cervical cancer (Andersson et al., 2000; Zehbe et al., 2001; Grodzki et al., 2006). However, the association was not consistently observed in other studies (Cornet et al., 2013b; Freitas et al., 2014). In our study, the EUR-350G pattern occurred at a similar rate, $67.5 \%$ in cervical cancers and $60 \%$ in low grade lesions and these differences were not statistically significant. Similar results in women with cervical cancer were observed in Slovenia (62.5\%) (Vrtačnik Bokal et al., 2010) but a lower frequency (49\%) of EUR 350G was reported across Europe in a recent meta-analysis (Tornesello et al., 2011). In our research the incidence of EUR-350G variant in the LSIL group was similar to the results of studies in Greece (64\%) and in Italy (65\%) but higher than in England (41\%) (Tsakogiannis et al., 2013; Burroni et al., 2013; Marongiu et al., 2014). In a recent study, the International Agency for Research on Cancer (IARC) HPV Variant Study Group revealed, by examining 1121 HPV16 positive cervical cancer cases and 400 HPV16 positive controls worldwide, that E$350 \mathrm{G}$ is associated with an increased risk of developing cervical cancer in South and Central America but not in Europe nor Central Asia (Cornet et al., 2013a). Furthermore, EUR-350T infections were more likely to persist and progress to CIN3 than in case of EUR-350G in Denmark (Gheit et al., 2011). Also Cornet and coworkers (2013b) demonstrated an approximately twofold risk of HPV16 persistence in the presence of EUR-350T. European variants with the polymorphism in EUR-350T were revealed to persist more often than those containing EUR350G (OR=1.6, 95\% CI=0.8-3.4).

In the previously conducted studies (Yamada et. al., 1997; Vrtačnik Bokal et al., 2010) additional nucleotide changes (T137G, G176A, T295G) occurring in EUR-350G were identified only in individual cases similarly as in our study. The frequencies of additional mutations (synonymous and non-synonymous) in EUR-350G variants were significantly higher than in EUR-350T in the present examination. These findings inspired us to try to assess the correlation between the presence of these additional mutations and clinical diagnosis and the physical status of HPV16 DNA. Additional mutations excluding T350G were observed 
in our samples more often in LSIL than in cervical cancer. Taking into consideration the relationship between the total number of nucleotide changes and the physical state of HPV16, we most frequently observed mutations in the mixed form of viral genome, while non-synonymous mutations were predominantly found in mixed and integrated states.

In our study, analysis of HPV16 E6 sequences showed three new (not found in the BLAST program) missense mutations associated with T350G in the LSIL (C153T, G122A) and cancer (G188A) group (Table 1). It seems that there is a large variability in HPV viruses circulating in the population affecting women with a low grade of cervical lesions, while in women with cancer, this variability is smaller. In the previous study we confirmed that together with the progression of changes in the direction of tumorigenesis, in women with multiple genotype HPV infection, there is a natural selection leading to infection with one genotype and it is usually HPV16 (Szostek et al., 2008a). Perhaps an analogical selection takes place also in case of mutated HPV16 strains along with the persistent infection and development of cancer.

Integration of the HPV genome in the host cell DNA represents a key step in the progression of cervical lesions, as it allows the continuous overexpression of E6 and E7 oncoproteins and its transforming activities via their interaction with $\mathrm{p} 53$ and $\mathrm{pRB}$, respectively (zur Housen, 2002). In the present study, the integrated HPV16 form was significantly more frequently detected in cervical cancer than in LSIL (Pearson's chi-squared $26.9(\mathrm{df}=2), p<0.0001)$. These results are consistent with other authors (Han et al., 2015; Shukla et al., 2014). In our previous study, the increase of frequency of the integrated HPV16 DNA with progression of dysplastic lesions of the cervix was shown (Szostek et. al., 2008b, Szostek et al., 2011). Some authors showed relatively high prevalence of mixed and integrated forms of HPV16 DNA in cervical epithelial cells obtained from women with low grade cervical lesions (Peisaro et al., 2002; Kumala et al., 2006; Huang et al., 2008). In our study, 72.5\% $(29 / 40)$ of samples with mixed form of HPV16 and one sample with integration of viral genome were detected in the LSIL group. In research of Huang and coworkers (2008) mixed form was detected in $83.3 \%(5 / 6)$ of samples while in Peisaro and coworkers (2002) - even in $93.3 \%(14 \mathrm{z} 15)$ but in CINI/II group. Furthermore, Kumala and coworkers (2006) identified dominant mixed form among women with persistent Pap smear abnormalities as well as among women who cleared their Pap smear abnormalities, $50 \%$ and $51.4 \%$, respectively. Our findings and the results of previous studies strongly indicate that the integration of HPV16 may occur in the early stages of neoplastic transformation of the cervix and could be a prognostic factor of cervical cancer.

We found a high incidence of the mixed form of HPV16 both in cervical cancer and in LSIL, but we observed that only in samples from cervical cancer with integrated form of HPV 16 genome two specific variants: EUR-350T and EUR-350G, without additional mutations dominated. However, it should be noted that the group analysed by us consisted of only 23 patients. It was also observed that non-synonymous and synonymous mutations were significantly more frequent in the mixed form of HPV 16 genome and in LSIL group.

In conclusion, the results of our study showed that, in the studied population of women from southern Poland, mainly European variants of HPV16 E6 could be found. The EUR-350G variant was very common in cervical cancer as well as in low grade lesions.
Interestingly, the additional nucleotide changes occurred significantly more often in $350 \mathrm{G}$ variants than in $350 \mathrm{~T}$ variants. Thus, we concluded that the presence of $350 \mathrm{G}$ mutation could affect the number of additional nucleotide changes appearing in the studied HPV16 E6 gene sequence, but this was mostly observed in LSIL.

Our study suggested that $350 \mathrm{~T}$ and $350 \mathrm{G}$ variants without additional mutations promoted viral integration to the host genome which can lead to cancer development. Therefore, it seems that the study of HPV16 E6 gene polymorphism may have prognostic significance in long-term monitoring of women.

\section{Acknowledgements}

This research was supported by KNOW Leading National Research Centre 2012 - 2017.

\section{REFERENCES}

Andersson S, Alemi M, Rylander E, Strand A, Larsson B, Sällström J, Wilander E (2000) Uneven distribution of HPV 16 E6 prototype and variant (L83V) oncoprotein in cervical neoplastic lesions. Br J Cancer 83: 307-310. doi: 10.1054/bjoc.2000.1247

Bernard HU, Burk RD, Chen Z, van Doorslaer K, Hausen H, deVilliers EM (2010) Classification of papillomaviruses (PVs) based on $189 \mathrm{PV}$ types and proposal of taxonomic amendments. Virology 401: 70-79. doi: 10.1016/j.virol.2010.02.002

Bruni L, Barrionuevo-Rosas L, Albero G, Aldea M, Serrano B, Valencia S, Brotons M, Mena M, Cosano R, Muñoz J, Bosch FX, de Sanjosé S, Castellsagué X. ICO Information Centre on HPV and Cancer (HPV Information Centre). Human Papillomavirus and Related Diseases in the World. Summary Report 2015, 12-23. http://www. hpvcentre.net/statistics/reports/XWX.pdf

Burk RD, Harari A, Chen Z (2013) Human papillomavirus genome variants. Virology 445: 232-243. doi: 10.1016/j.virol.2013.07.018

Burroni E, Bisanzi S, Sani C, Puliti D, Carozzi F (2013) Codon 72 polymorphism of p53 and HPV type $16 \mathrm{E} 6$ variants as risk factors for patients with squamous epithelial lesion of the uterine cervix.J Med Virol 85: 83-90. doi: 10.1002/jmv.23417

Cornet I, Gheit T, Franceschi S, Vignat J, Burk RD, Sylla BS, Tommasino M, Clifford GM (2012) Human papillomavirus type 16 genetic variants: phylogeny and classification based on E6 and LCR. IARC HPV Variant Study Group. J Virol 86: 6855-6861. doi:10.1128/ JVI.00483-12

Cornet I, Gheit T, Iannacone MR, Vignat J, Sylla BS, Del Mistro A, Franceschi S, Tommasino M, Clifford GM (2013a) HPV16 genetic variation and the development of cervical cancer worldwide. $\mathrm{Br} J$ Cancer 108: 240-244. doi: 10.1038/bjc.2012.508

Cornet I, Gheit T, Clifford GM, Combes JD, Dalstein V, Franceschi S, Tommasino M, Clavel C (2013b) Human papillomavirus type 16 E6 variants in France and risk of viral persistence. Infect Agent Cancer 8: 4. doi: 10.1186/1750-9378-8-4

Crow JM. (2012) HPV: the global burden. Nature 488: S2-S3. doi: 10.1038/488S2a

de Araujo Souza PS, Sichero L, Maciag PC (2009) HPV variants and HLA polymorphisms: the role of variability on the risk of cervical cancer. Future Oncol 5: 359-370. doi: 10.2217/fon.09.8

de Villiers EM, Fauquet C, Broker TR, Bernard HU, zurHausen H (2004) Classification of papillomaviruses. Virology 324: 17-27. doi: 10.1002/(SICI)1097-0215(19961021)69:5<364: :AID-IJC2>3.0.CO;2-3

Freitas LB, Chen Z, Muqui EF, Boldrini NA, Miranda AE, Spano LC, Burk RD (2014) Human papillomavirus 16 non-European variants are preferentially associated with high-grade cervical lesions. PLoS One 9: e100746. doi: 10.1371/journal.pone.0100746

Garbuglia AR. (2014) Human papillomavirus in head and neck cancer. Cancers 6: 1705-1726. doi: 10.3390/cancers6031705

Gheit T, Cornet I, Clifford GM, Iftner T, Munk C, Tommasino M, Kjaer SK (2011) Risks for persistence and progression by human papillomavirus type 16 variant lineages among a population-based sample of Danish women.Cancer Epidemiol Biomarkers Prev 20: 13151321. doi: 10.1158/1055-9965.EPI-10-1187

Grodzki M, Besson G, Clavel C, Arslan A, Franceschi S, Birembaut P, Tommasino M, Zehbe I (2006) Increased risk for cervical disease progression of French women infected with the human papillomavirus type 16 E6-350G variant. Cancer Epidemiol Biomarkers Prev 15: 820-822. doi: 10.1158/1055-9965.EPI-05-0864

Gudlevičienè Ž, Stumbrytė A, Juknè G, Simanavičienè V, Žvirblienè A (2015) Distribution of human papillomavirus type 16 variants in Lithuanian women with cervical cancer. Medicina (Kaunas) 51: 328335. doi: 10.1016/j.medici.2015.11.005 
Han L, Maimaitiming T, Husaiyin S, Wang L, Wusainahong K, Ma C, Niyazi M (2015) Comparative study of HPV16 integration in cervical lesions between ethnicities with high and low rates of infection with high-risk HPV and the correlation between integration rate and cervical neoplasia. ExpTher Med 10: 2169-2174. doi: 10.3892/ etm.2015.2740

Hildesheim A, Schiffman M, Bromley C, Wacholder S, Herrero R, Rodriguez A, Bratti MC, Sherman ME, Scarpidis U, Lin QQ, Terai M, Bromley RL, Buetow K, Apple RJ, Burk RD (2001) Human papillomavirus type 16 variants and risk of cervical cancer. $J$ Natl Cancer Inst 93: 315-318. doi: 10.1093/jnci/93.4.315

Huang L, Chao S, Lee B (2008) Integration of human papillomavirus type-16 and type-18 is a very early event in cervical carcinogenesis. $J$ ClinPathol 61: 627-631. doi: 10.1136/jcp.2007.052027

Kulmala SM, Syrjänen SM, Gyllensten UB, Shabalova IP, Petrovichev N, Tosi P, Syrjänen KJ, Johansson BC (2006) Early integration of high copy HPV16 detectable in women with normal and low grade cervical cytology and histology. I ClinPathol 59: 513-517. doi: 10.1136/jcp. 2004.024570

Lee K, Magalhaes I, Clavel C, Briolat J, Birembaut P, Tommasino M, Zehbe I (2008) Human papillomavirus 16 E6, L1, L2 and E2 gene variants in cervical lesion progression. Virus Res131: 106-110. doi: 10.1016/j.virusres.2007.08.003

Londesborough P, Ho L, Terry G, Cuzick J, Wheeler C, Singer A (1996) Human papillomavirus genotype as a predictor of persistence and development of high-grade lesions in women with minor cervical abnormalities. Int J Cancer 21: 364-368

Marongiu L, Godi A, Parry JV, Beddows S (2014) Human papillomavirus type 16 long control region and E6 variants stratified by cervical disease stage. Infect Genet Evol 26: 8-13. doi: 10.1016/j. meegid.2014.05.009

Muñoz N, Bosch FX, de Sanjosé S, Herrero R, Castellsagué X, Shah KV, Snijders PJ, Meijer CJ (2003) International Agency for Research on Cancer Multicenter Cervical Cancer Study Group. Epidemiologic classification of human papillomavirus types associated with cervical cancer. $N$ Engl J Med 348: 518-527. doi: 10.1056/NEJMoa021641

Nindl I, Rindfleisch K, Lotz B, Schneider A, Dürst M (1999) Uniform distribution of HPV $16 \mathrm{E} 6$ and E7 variants in patients with normal histology, cervical intra-epithelial neoplasia and cervical cancer. Int J Cancer 82: 203-207. doi: 10.1002/(SICI)1097-0215(19990719)82:2 $<203:$ :AID-IJC9>3.0.CO;2-9

Ortiz-Ortiz J, Alarcón-Romero Ldel C, Jiménez-López MA, GarzónBarrientos VH, Calleja-Macías I, Barrera-Saldaña HA, LeyvaVázquez MA, Illades-Aguiar B (2015) Association of human papillomavirus 16 E6 variants with cervical carcinoma and precursor lesions in women from Southern Mexico. Virol J 12: 29. doi: 10.1186/s12985-015-0242-3

Peitsaro P, Johansson B, Syrjänen S (2002) Integrated human papillomavirus type 16 is frequently found in cervical cancer precursors as demonstrated by a novel quantitative real-time PCR technique. J Clin Microbiol 40: 886-891. doi: 10.1128/JCM.40.3.886-891.2002

Schiffman M, Rodriguez AC, Chen Z, Wacholder S, Herrero R, Hildesheim A, Desalle R, Befano B, Yu K, Safaeian M, Sherman ME, Morales J, Guillen D, Alfaro M, Hutchinson M, Solomon D, Castle PE, Burk RD (2010) A population-based prospective study of carcinogenic human papillomavirus variant lineages, viral persistence, and cervical neoplasia. Cancer Res 70: 3159-3169. doi: 10.1158/0008-5472.CAN-09-4179

Shukla S, Mahata S, Shishodia G, Pande S, Verma G, Hedau S, Bhambhani S, Kumari A, Batra S, Basir SF, Das BC, Bharti AC (2014) Physical state \& copy number of high risk human papillomavirus type 16 DNA in progression of cervical cancer. Indian J Med Res 139: 531-543

Sichero L, Ferreira S, Trottier H, Duarte-Franco E, Ferenczy A, Franco EL, Villa LL (2007) High grade cervical lesions are caused preferentially by non-European variants of HPVs 16 and 18. Int J Cancer 120: 1763-1768. doi: 10.1002/ijc.22481

Sichero L, Sobrinho JS, Villa LL (2012) Oncogenic potential diverge among human papillomavirus type 16 natural variants. Virology 432: 127-132. doi: 10.1016/j.virol.2012.06.011
Szostek S, Klimek M, Zawilinska B, Kosz-Vnenchak M (2008a) Genotype-specific human papillomavirus detection in cervical smears. ActaBiochim Pol 55: 687-692

Szostek S, Klimek M, Zawilinska B, Kosz-Vnenchak M (2008b) Physical state of human papillomavirus type 16 in cervical cell DNA. Folia Biol (Krakow) 56: 269-271. doi:10.3409/fb.56_3-4.269-271

Szostek S, Zawilińska B, Klimek M, Wójcik K, Koprynia M, KoszVnenchak M (2011) Differentiation of an integrated and episomal HPV-16 DNA using real-time PCR in cervical specimens of women diagnosed with intraepithelial lesions and invasive cervical cancer. Ginekol Pol 82: 441-445 (in Polish)

Szostek S, Biesaga B, Zawilinska B, Klimek M, Kosz-Vnenchak M (2015) Physical state of human papillomavirus type 16 in cervical intraepithelial lesions and cancers determined by two different quantitative real-time PCR methods. Acta Biochim Pol 62: 923-928. doi: 10.18388/abp.2015_1161

Tornesello ML, Losito S, Benincasa G, Fulciniti F, Botti G, Greggi S, Buonaguro L, Buonaguro FM (2011) Human papillomavirus (HPV) genotypes and HPV16 variants and risk of adenocarcinoma and squamous cell carcinoma of the cervix. Gynecol Oncol 121: 32-42. doi: 10.1016/j.ygyno.2010.12.005

Tsakogiannis D, Papadopoulou A, Kontostathi G, Ruether IG, Kyriakopoulou Z, Dimitriou TG, Orfanoudakis G, Markoulatos P (2013) Molecular and evolutionary analysis of HPV16 E6 and E7 genes in Greek women. J Med Microbiol 62: 1688-1696. doi:10.1099/ imm.0.055491-0

van Duin M, Snijders PJ, Vossen MT, Klaassen E, Voorhorst F, Verheijen RH, Helmerhorst TJ, Meijer CJ, Walboomers JM (2000) Analysis of human papillomavirus type $16 \mathrm{E} 6$ variants in relation to p53 codon 72 polymorphism genotypes in cervical carcinogenesis. $J$ Gen Virol 81: 317-325. doi:10.1159/000016735

VrtačnikBokal E, Kocjan BJ, Poljak M, Bogovac Z, Jančar N (2010) Genomic variants of human papillomavirus genotypes 16, 18, and 33 in women with cervical cancer in Slovenia. J Obstet Gynaecol Res 36: 1204-1213. doi: 10.1111/j.1447-0756

Walboomers, JMM., Jacobs MV, Manos MM, Bosch FX, Kummer JA, Shah KV, Snijders PJF, Peto J, Meijer CJLM, Munoz N (1999) Human papillomavirus is a necessary cause of invasive cervical cancer worldwide. I Pathol 189: 12-19. doi: 10.1002/(SICI)10969896(199909)189:1<12::AID-PATH431>3.0.CO;2-F

Xi LF, Kiviat NB, Hildesheim A, Galloway DA, Wheeler CM, Ho J, Koutsky LA (2006) Human papillomavirus type 16 and 18 variants: race-related distribution and persistence. J Natl Cancer Inst 98: 10451052. doi: $10.1093 /$ jnci/dji 297

Xi LF, Koutsky LA, Hildesheim A, Galloway DA, Wheeler CM, Winer RL, Ho J, Kiviat NB (2007) Risk for high-grade cervical intraepithelial neoplasia associated with variants of human papillomavirus types 16 and 18. Cancer Epidemiol Biomarkers Prev 16: 4-10. doi: 10.1158/1055-9965.EPI-06-0670

Yamada T, Manos MM, Peto J, Greer CE, Munoz N, Bosch FX, Wheeler CM (1997) Human papillomavirus type 16 sequence variation in cervical cancers: a worldwide perspective. J Virol 71: 24632472. 0022-538X/97/\$04.0010

Zehbe I, Wilander E, Delius H, Tommasino M (1998) Human papillomavirus 16 E6 variants are more prevalent in invasive cervical carcinoma than the prototype.Cancer Res 58: 829-833. http://cancerres. aacrjournals.org/content/58/4/829

Zehbe I, Tachezy R, Mytilineos J, Voglino G, Mikyskova I, Delius H, Marongiu A, Gissmann L, Wilander E, Tommasino M (2001) Human papillomavirus $16 \mathrm{E} 6$ polymorphisms in cervical lesions from different European populations and their correlation with human leukocyte antigen class II haplotypes. Int J Cancer 94: 711-716. doi: $10.1002 /$ ijc. 1520

Zuna RE, Moore WE, Shanesmith RP, Dunn ST, Wang SS, Schiffman M, Blakey GL, Teel T (2009) Association of HPV16 E6 variants with diagnostic severity in cervical cytology samples of 354 women in a US population. Int J Cancer 125: 2609-2613. doi: 10.1002/ ijc. 24706

zurHausen H (2002) Papillomaviruses and cancer: from basic studies to clinical application. Nat Rev Cancer 2: 342-350. doi:10.1038/nrc798 